\title{
2015 Proceedings
}

Assessing antibacterial property, UV protection, and thermal comfort of nanosilver treated cotton and polyester knit fabrics for sportswear

Rui Li, Iowa State University, USA

Jie Yang, Tsinghua University, China

Chunhui Xiang, Iowa State University, USA

Keywords: nanosilver, antibacterial, thermal comfort, dynamic thermal model

Introduction and literature review The development of nanotechnology has added many functions such as antibacterial property and UV protection to sportswear (Ozdil \& Anand, 2014). Among a variety of treatment to the fabrics, nanosilver can provide great antibacterial property without harming the wearer or the environment, which in turn enhances the hygiene and odor control ability for the sportswear (Saad \& Hafez, 2014). In addition, the incorporation of metal nanoparticles like silver can also increase the UV protection ability for the sportswear, which is highly desirable for outdoor activities. However, sportswear is expected to provide both good performance and comfort for the wearer. Thermal comfort is crucially important for sportswear because the excessive heat generated in sport activities need to be effectively and efficiently transferred out to achieve thermal balance (Ozdil \& Anand, 2014). Although many studies have validated the antibacterial property of nanosilver treated fabrics (e.g. Paladini, De Simone, Sannino, \& Pollini, 2014), few has been done on the assessment of UV protection property and/or thermal comfort. No study has been found assessing thermal comfort of nanosilver treated fabrics with human, clothing, and environment all taken into consideration. Hence, the purpose of this study was to introduce a dynamic numerical thermal model to assess the thermal comfort of nanosilver treated knit fabrics for sportswear. The antibacterial property and UV protection of the nanosilver treated knit fabrics were also evaluated.

Materials and treatment Plain cotton and polyester jersey knit fabrics were for this study because of their wide usage in sportswear. Three nanosilver solutions were obtained by dissolving respectively $0.1 \mathrm{wt} / \mathrm{v} \%, 0.5 \mathrm{wt} / \mathrm{v} \%$, and $1 \mathrm{wt} / \mathrm{v} \%$ of silver nitrate in a mixture of $10 \mathrm{v} / \mathrm{v} \%$ methanol and deionized water. Cotton and polyester fabrics were dip coated in these silver solutions and then immediately exposed to UV radiation for $20 \mathrm{~min}$ in order to induce the photoreduction of the silver precursor and the formation of silver clusters on the surface of the fabrics (Paladini et al., 2014). Then, the fabrics were washed with water to remove unreacted salt and dried in an oven at 50C for 1 hour.

Experiments and analysis Scanning electron microscopy (SEM) was used to study the morphology and the distribution of the silver nanoparticles on surface of the fabrics. Energy dispersive X-ray spectroscopy (EDS) was used to confirm the presence of silver on the surface of fabrics. UV-Vis spectrophotometer was used to analyze the transmission of UV radiation through the fabrics. An agar diffusion test with $E$. Coli was conducted to assess the antibacterial property of the materials (Paladini et al., 2014). Thermal resistance (Rct) and evaporate resistance (Ret) of the fabrics were measured using a sweat guarded hot plate. The Rct and Ret results were input into a human thermal model to simulate human body temperature with

Page 1 of 2

(c) 2015, International Textile and Apparel Association, Inc. ALL RIGHTS RESERVED ITAA Proceedings, \#72 - www.itaaonline.org 
metabolism (M), air temperature (T), relative humidity $(\mathrm{RH})$, and wind speed $(\mathrm{V})$ taken into account (Yang, Weng, \& Zhang, 2014). The human thermal model was then coupled with a thermal sensation index (TSENS) with 11-point scale as shown in Fig 1. (Zolfaghari \& Maerefat, 2011). Thus, the thermal comfort of the fabrics was assessed dynamically by computer simulation in four different temperatures with one hour time span.

Results and conclusions The SEM and EDS test confirmed successful application of silver nanoparticles on cotton and polyester knit fabrics with different concentrations of silver solution. UV-vis test results showed considerable reduction of UV transmission through treated fabrics. However, no obvious differences were found among different solution concentrations. Both treated cotton and polyester fabrics showed observable uninhabited zone in the agar diffusion test regardless of the silver solution concentration, indicating satisfactory antibacterial property. The thermal resistance for cotton fabrics was not significantly changed after applying nanosilver, probably because cotton itself as one natural fiber already has good thermal conductivity. However, the treated polyester fabrics showed significantly lowered thermal resistance compared with untreated one. The reason could be the silver nanoparticles increased surface thermal conductivity on polyester. As a result, the human thermal model predicts that the treated polyester fabrics have different thermal sensation for the wearer in a sport activity compared with untreated one (e.g. Fig 2.). This study demonstrated that adding nanosilver to cotton and polyester fabrics can offer desirable antibacterial property and UV protection without significantly affecting their thermal properties. A dynamic numeric model was introduced for assessing fabrics thermal comfort in a human-clothing-environment system for the first time. Future research is needed for the validation of the results of this study using human trial.

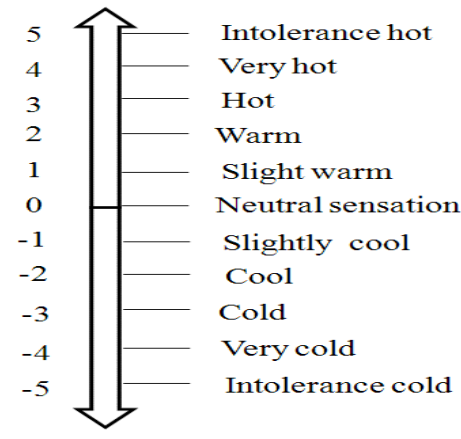

Fig 1. The 11-points scale of TSENS

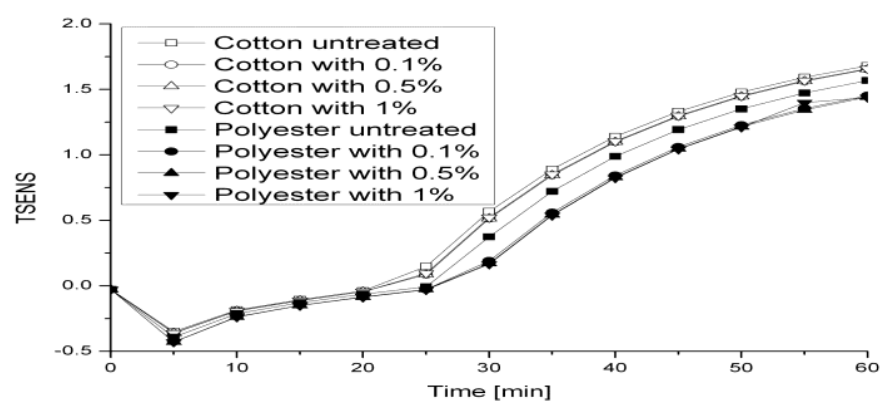

Fig 2. The values of the TSENS in temperature of $15^{\circ} \mathrm{C}$

Reference

Ozdil, N., \& Anand, S. (2014). Recent developments in textile materials and products used for activewear and sportswear. Tekstil Teknolojileri Elektronik Dergisi, 8(3), 68-83.

Paladini, F., De Simone, S., Sannino, A., \& Pollini, M. (2014). Antibacterial and antifungal dressings obtained by photochemical deposition of silver nanoparticles. Journal of Applied Polymer Science, 131(11), 40326.

Saad, E. R., \& Hafez, N. M. (2014). Effect of coating with silver nanoparticles (AgNPs) on cotton fabric functional properties. International Design Journal, 4(2), 33-39.

Yang, J., Weng, W., \& Zhang, B. (2014). Experimental and numerical study of physiological responses in hot environments. Journal of thermal biology, 45, 54-61.

Zolfaghari, A., \& Maerefat, M. (2011). A new predictive index for evaluating both thermal sensation and thermal response of the human body. Building and Environment, 46(4), 855-862.

Page 2 of 2

(C) 2015, International Textile and Apparel Association, Inc. ALL RIGHTS RESERVED ITAA Proceedings, \#72 - www.itaaonline.org 